\section{hommes}

\section{Hommes \& migrations}

Revue française de référence sur les dynamiques

migratoires

1307 | 2014

L'Afrique qualifiée dans la mondialisation

\title{
La diaspora sénégalaise au Canada
}

\section{Magatte Fall}

\section{(2) OpenEdition}

Journals

\section{Édition électronique}

URL : http://journals.openedition.org/hommesmigrations/2871

DOI : 10.4000/hommesmigrations.2871

ISSN : 2262-3353

\section{Éditeur}

Musée national de l'histoire de l'immigration

\section{Édition imprimée}

Date de publication : 1 juillet 2014

Pagination : 9-16

ISBN : 978-2-919040-28-5

ISSN : $1142-852 X$

\section{Référence électronique}

Magatte Fall, « La diaspora sénégalaise au Canada », Hommes \& migrations [En ligne], 1307 | 2014, mis en ligne le 01 juillet 2017, consulté le 01 mai 2019. URL : http://journals.openedition.org/ hommesmigrations/2871; DOI : 10.4000/hommesmigrations.2871 


\section{LA DIASPORA SÉNÉGALAISE AU CANADA}

par MAGATTE FALL, docteur en géographie, membre du réseau REDTAC (Im) migration, université de Montréal.

Face aux obstacles qu'elles rencontrent sur le marché du travail européen, les personnes hautement qualifiées originaires du Sénégal choisissent de tenter leur chance outre-Atlantique. Depuis une dizaine d'années, le Canada est devenu une destination prisée par les diplômés sénégalais. Si l'installation dans ce pays s'avère relativement aisée, il n'en va pas de même pour trouver un emploi en accord avec sa formation. La société canadienne demeure accueillante, mais s'y faire une place demande du temps.

Définir la diaspora sénégalaise n'est pas chose facile. Le concept de diaspora désigne non seulement la dispersion d'une communauté ethnique ou d'un peuple à travers le monde, mais aussi le résultat de cette dispersion, c'est-à-dire l'ensemble des membres d'une communauté dispersés dans plusieurs pays. Ainsi, les problématiques qui touchent les diasporas concernent l'espace économique, les flux transnationaux, les structures religieuses, les comparaisons entre les différents modes d'accueil des pays récepteurs, la notion de territorialité et les grands espaces carrefours.

Afin de mieux cerner les enjeux de la migration internationale sénégalaise, cet article est consacré à la diaspora basée au Canada. La plupart des données statistiques utilisées ici sont extraites des bases de données de Statistique Canada, notamment le recensement de la population de 2006, et de données de Citoyenneté Immigration Canada (CIC). Notre étude concerne surtout les Sénégalais qui ont un statut de résident permanent au Canada. Les personnes ayant un statut de migrant temporaire ont été exclues de cette recherche en raison de la brièveté de leur séjour qui ne permet pas une étude approfondie. La particularité de cette diaspora sénégalaise est d'être majoritairement constituée de personnes hautement qualifiées, ce qui laisse croire à "une fuite des cerveaux" dans un pays où les défis du développement sont plus que présents. 


\section{Le problème du retour des étudiants sénégalais}

La question du retour a toujours constitué un problème pour la migration sénégalaise. Dans une chronique consacrée aux migrations soninkés, Monique Chastenet le souligne: "François Manchuelle note dans sa conclusion que le paradoxe des migrations temporaires est qu'elles évoluent vers une situation où le retour du migrant devient de plus en plus problématique. Les migrations soninkées en France en sont là, après une phase d'essor dans les années 19601970. Les années 1980 sont marquées par le développement du regroupement familial et le quasi-abandon des migrations tournantes au sein d'une même famille ${ }^{1}$." C'est cette même situation migratoire, en raison de son caractère temporaire, qui affecte depuis quelques

Le Canada est

officiellement devenu l'un des pays qui accueillent le plus de "personnes hautement qualifiées" dans le monde. décennies les étudiants sénégalais diplômés de l'étranger.

$\mathrm{Si}$, pendant la colonisation et après l'indépendance, jusqu'aux années 1980, la majeure partie retournaient au Sénégal pour leur insertion, cela n'est plus le cas aujourd'hui ${ }^{2}$. Le Sénégal est frappé par un taux de chômage élevé. Il n'existe pas de structures capables de favoriser le retour et l'insertion des étudiants diplômés à l'étranger. Parmi les étudiants qui choisissent le retour, ceux qui ont des réseaux facilitant leur insertion socioprofessionnelle arrivent aisément à trouver du travail. Ceux qui ne disposent pas de tels réseaux, après quelques jours voire quelques mois de recherches infructueuses, repartent dans leur pays de formation ou dans un autre pays du Nord. Ainsi, cette dernière catégorie va alimenter le phénomène de la "fuite des cerveaux", que d'autres chercheurs appellent "la circulation des compétences", dans la mesure où ce phénomène ne touche pas uniquement les pays en voie de développement, comme l'expliquent la plupart des ouvrages qui traitent de la migration des personnes hautement qualifiées. Le Sénégal devrait se doter de moyens pour encourager le retour des intellectuels, comme ont bien réussi à le faire les pays de l'Asie du Sud-Est, en favorisant la connexion de ses intellectuels basés à létranger et ceux restés au pays, par le biais de conférences, de symposiums et de séminaires. Il est également nécessaire de préparer les étudiants qui sont à l'étranger à un retour au pays, en organisant des forums d'emploi, en créant des structures pour les accueillir, ppour les aider à chercher un emploi, à se préparer à l'entretien d'embauche, à intégrer le système de "réseautage", etc.

\section{Un pays attractif pour l'immigration des hauts potentiels}

Le Canada est officiellement devenu l'un des pays qui accueillent le plus de "personnes hautement qualifiées" dans le monde. En effet, l'enquête nationale auprès des ménages (ENM) de 2011, publiée par Statistique Canada, mentionne que 1 Canadien sur 5 est né à létranger, soit 6775800 personnes. Les raisons de son attrait sont diverses. Le Canada est une terre d'accueil. De par sa tradition et sa politique, ce pays a toujours encouragé la diversité culturelle. Deuxième pays au monde en terme de superficie, le Canada, composé de dix provinces et de trois territoires, dispose d'un environnement magnifique. C'est un pays reconnu pour être une société sûre, paisible et juste, qui offre une éducation de qualité. Il est classé parmi les premiers pays de l'Organisation de coopération et de développement économiques (OCDE) pour la promotion au niveau des études postsecondaires. Le Canada est également cité parmi les pays ayant la meilleure

1. Monique Chastenet, “Les migrations soninkées dans la longue durée : stratégies et identités", in Cahiers d'études africaines, vol. 39 , $n^{\circ} 153,1999$, p. 173 2. Magatte Fall, "La migration des étudiants sénégalais en France : ses déterminants, les conditions de séjour des étudiants et leur devenir", thèse de doctorat de géographie, Lille, université de Lille-I, 2009. 3. Anne Marie Gaillard, Jacques Gaillard, Les Enjeux des migrations scientifiques internationales. De la quête du savoir à la circulation des compétences, Paris, L’Harmattan, 1999. 
Pollack gère la plus ancienne auto-école installée sur

le "campus ancien" de Cocody. L'inscription au permis de conduire coûte officiellement 90 ooo francs CFA (140 euros).

Abidjan, mars 2010. @ CAMILLE MILLERAND

qualité de vie, en fonction de certains critères comme l'espérance de vie, le niveau de l'instruction et le revenu par habitant, mais aussi la gratuité des soins de santé, un régime de sécurité sociale et la garantie des droits et des libertés aux citoyens. C'est, enfin, un territoire propice aux affaires : en plus de ses vastes ressources, il est le plus important partenaire commercial des États-Unis et entretient d'excellentes relations avec d'autres pays sur le continent américain et ailleurs dans le monde.

Cependant, le processus de sélection des candidats à l'immigration régulière se fait à travers un système coûteux et long pour les migrants. Le gouvernement du Canada, en collaboration avec les provinces, les territoires et les principaux intervenants, établit le nombre d'immigrants qui seront admis dans le pays chaque année. Au cours des dix dernières années, le Canada a accueilli en moyenne 220770 immigrants et réfugiés par année. Ainsi, le Canada reconnaît les avantages à attirer les travailleurs hautement qualifiés. Près de $59 \%$ des immigrants de ce pays font partie de cette catégorie. Ils aident à combler les pénuries de main-d'œuvre sur le marché du travail ou investissent dans l'économie. Selon l'ENM de 2011, la part des ressortissants africains est de $12,5 \%$, avec une légère augmentation d'environ $2 \%$ des ressortissants venant de l’Algérie, du Maroc et du Nigeria.

Face aux différentes destinations des Sénégalais à l'étranger, le Canada est le pays préféré des étudiants 
sénégalais diplômés. On constate une réelle augmentation du flux migratoire en direction de ce pays. De 2005 à 2010, l'effectif des Sénégalais ayant un statut de résident permanent au Canada est passé de 117 à 465 selon les statistiques de Citoyenneté et Immigration Canada. Cette migration est constituée d'anciens étudiants qui ont fait le choix de rester à la fin de leurs études et d'une nouvelle vague d'immigrants venus de France et d'autres pays d'Europe occidentale (généralement d'anciens étudiants) qui, faute de pouvoir s'installer dans leur pays de formation, ont décidé de poursuivre leur migration vers le Canada.

\section{Migrer au Canada après un détour par l'Europe}

La venue des Sénégalais au Canada date des années 1980. Le Canada accueillait des intellectuels sénégalais, notamment dans le cadre deprogrammes d'exonération de frais d'inscription et de bourses pour les étudiants. La classe bourgeoise sénégalaise avait l'habitude d'en-

Contrairement voyer ses enfants dans les uniau renouvellement des titres de séjour qui détermine

la possibilité de travailler en Europe, au Canada, dès la réception d'un numéro

d'assurance sociale, le marché

du travail canadien est complètement ouvert.

le territoire canadien (voir tableau $n^{\circ} 1$ ci-contre)

Ce départ s'explique par les difficultés que rencontrent les étudiants sénégalais à la fin de leurs études en Europe, liées à leur changement de statut et à l'obtention d'une carte de séjour, de travail. La signature de conventions avec les pays d'origine oblige les étudiants à retourner dans leur pays une fois leurs diplômes obtenus. Par ailleurs, face au chômage croissant en Europe, la plupart des étudiants sénégalais font des études dans des disciplines générales, sans spécialisation (sciences sociales, sciences économiques, sciences juridiques, etc.). Or le marché européen n'est plus ouvert comme avant à de telles formations.

Enfin, il faut mentionner la crainte pour les Sénégalais de retourner dans leur pays d'origine. Le Sénégal est affecté par un taux important de chômage chez les jeunes diplômés. Selon l'étude de la Direction des statistiques sur l'emploi des jeunes au Sénégal publiée en juin 2013, "le taux d'emploi des jeunes au Sénégal est resté stable pour la période de 2005-2011 en dépit d'un recul de 2 points. En effet, il tourne autour de $38 \%$. Or, en 2011, le taux d'activité des jeunes est passé à $42 \%$, contre 44,4\% en 2005 . Paradoxe, ce sont les plus diplômés qui présentent les taux d'activité les plus faibles avec un pourcentage de $20 \%$ pour le niveau secondaire et $36 \%$ pour le niveau supérieur".".

Pour ces diplômés sénégalais, le Canada est un pays rêvé où il est possible dès l'arrivée d'obtenir une carte de résidence permanente et, après un séjour de trois ans, de pouvoir faire la demande pour être citoyen canadien. Contrairement au renouvellement des titres de séjour qui détermine la possibilité de travailler en Europe, au Canada, dès la réception d'un numéro d'assurance sociale, le marché du travail canadien est complètement ouvert.

\section{Analyse de la croissance et de la répartition spatiale de la migration sénégalaise au Canada}

Les Sénégalais immigrants au Canada sont constitués en majorité de personnes hautement qualifiées : plus de la moitié d'entre eux ont un niveau égal ou supérieur à un master (bac +5$)$.

D'après le tableau n ${ }^{\circ} 1$, nous constatons une croissance de la population sénégalaise obtenant chaque année un statut de résident permanent au Canada. En 2002 et 2004, nous remarquons une légère 
chute. Cependant, à partir de 2005, les effectifs de la population sénégalaise au Canada augmentent. À l'arrivée de forts contingents de jeunes diplômés sénégalais en provenance d'Europe, il faut ajouter celle d'immigrants venus directement du Sénégal, à partir de 2008, dans le cadre du programme canadien d'immigration de personnes hautement qualifiées, avec la création à Dakar d'agences privées chargées de promouvoir la venue au Canada de diplômés et de cadres sénégalais (Accès Canada). Sans oublier l'immigration pour regroupement familial qui connaît un essor important. Les étudiants sénégalais diplômés qui s'insèrent dans le tissu économique canadien sont directement répertoriés dans la catégorie des personnes hautement qualifiées venues s'installer au Canada. C'est pourquoi, dans cet article, nous avons davantage utilisé les statistiques de la communauté sénégalaise (immigrante) au Canada que celles d'étudiants diplômés venus de l'extérieur.

/// Tableau nº 1: L'évolution du nombre de Sénégalais obtenant chaque année une carte de résident permanent au Canada.

\begin{tabular}{|c|c|}
\hline Années & Effectifs \\
\hline 2001 & 117 \\
\hline 2002 & 95 \\
\hline 2003 & 182 \\
\hline 2004 & 152 \\
\hline 2005 & 205 \\
\hline 2006 & 208 \\
\hline 2007 & 365 \\
\hline 2008 & 386 \\
\hline 2009 & 454 \\
\hline 2010 & 465 \\
\hline
\end{tabular}

Source : extrait du tableau Canada-résidence permanente selon les pays d'origine (CIC).

La majeure partie des Sénégalais sont installés au Québec, attirés par le caractère francophone de la province. Selon le recensement de 2006 de
Statistique Canada, la province du Québec est la préférée des Sénégalais avec un effectif de 2 605, devant la province de l'Ontario (305), le Manitoba (155), l'Alberta (35), le Nouveau-Brunswick (30) et la Colombie-Britannique (20). Les étudiants sénégalais diplômés sont davantage attirés par une province qui promet une insertion plus facile dans un pays où l'anglais est la langue dominante. Depuis septembre 2008, une filière post-diplôme s'adresse aux travailleurs étrangers temporaires souhaitant s'établir au Québec. L’une des exigen-ces est d'avoir une expé-
Le Canada a défini une politique de quotas en matière d'immigration qui lui permet de contrôler les flux migratoires. rience de travail de vingt-quatre mois au Canada à un niveau de compétences élevé durant les trois ans précédant la demande, de même que de bonnes aptitudes linguistiques en anglais ou en français.

\section{Les différents programmes d'immigration au Canada}

Le Canada a défini une politique de quotas en matière d'immigration qui lui permet de contrôler les flux migratoires. Si chaque province a sa politique de sélection des immigrants, il existe un programme concernant les travailleurs qualifiés au niveau du gouvernement fédéral, signé par la majeure partie des provinces et territoires canadiens. C'est le cas des provinces et territoires suivants : Alberta, Île-du-Prince-Édouard, NouveauBrunswick, Ontario, Terre-Neuve et Labrador, Colombie-Britannique, Manitoba, Nouvelle-Écosse, Saskatchewan, Yukon. L'accord signé entre les provinces et le gouvernement fédéral consiste à permettre aux premières de sélectionner des immigrants qui souhaitent s'installer sur leur territoire. Pour cela, il faut soumettre une demande à la province ou au territoire qui l'évaluera en fonction de ses besoins en matière d'immigration et de l'intention du demandeur à s'y établir de manière durable. 
Après avoir été accepté par une province ou territoire, on peut faire une demande de résidence permanente à Citoyenneté Immigration Canada (CIC) qui, à son tour, l'évaluera en fonction de la réglementation de l'immigration canadienne. Un des traits dominants de la migration sénégalaise au Canada est la quasi-parité entre les sexes. Les femmes occupent une part importante dans les effectifs de la population sénégalaise présente au Canada : environ 1 385, contre 1735 pour les hommes ${ }^{5}$. Cette importance est due en partie au nombre d'étudiantes qui arrivent au Canada et décident de changer de statut à la fin de leurs études, mais aussi à celles qui viennent dans le cadre du regroupement familial. Par rapport aux autres pays d'accueil, le Canada et les États-Unis sont en tête pour l'octroi de visas d'études aux étudiantes sénégalaises.

Le Canada a su faire du multiculturalisme sa force en matière d'immigration. Aujourd'hui, on trouve une multitude de communautés culturelles et chaque groupe revendique ses droits en vue de bien s'intégrer au Canada. Le gouvernement fédéral est le seul organisme à octroyer le droit de séjour. Cependant, en fonction des demandes de maind'œuvre dans certains secteurs d'activité, les gouvernements provinciaux, soutenus par les employeurs, peuvent faire pression afin de pousser le gouvernement fédéral à ouvrir le marché du travail à certaines catégories de travailleurs. C'est souvent le cas de la province du Québec.

\section{Les difficultés d'insertion des étudiants diplômés sénégalais}

Si l'installation au Canada en tant que résident permanent est relativement aisée pour les diplômés sénégalais, il n'en va pas de même pour leur insertion professionnelle.
La nécessité de recommencer une formation universitaire, le manque d'expérience canadienne et l'importance de l'anglais font partie des difficultés rencontrées. De manière générale, l'insertion au Canada se fait dans la durée. Il faut compter au minimum cinq ans avant de rentrer dans le moule canadien en passant par une petite formation dans une université ou un collège canadien. En 2006, les nouveaux immigrants étaient presque deux fois plus susceptibles d'avoir un diplôme universitaire que les personnes nées au Canada. Malgré cet avantage incontestable, un rapport de Statistique Canada stipule qu' "au cours de la période allant de 1991 à 2006, la proportion d'immigrants détenteurs d'un diplôme universitaire qui occupent des postes nécessitant un faible niveau scolaire (tels que commis, routier, vendeur, caissier ou chauffeur de taxi) a augmenté ${ }^{6}$. Une formation étrangère ne permet pas d'intégrer le marché du travail canadien aussi facilement qu'on le souhaiterait.

Il faut souligner que les diplômes étrangers ne sont pas bien connus des employeurs canadiens. Des organismes, comme le ministère de l'Immigration et des Communautés culturelles du Québec mais aussi le World Education Services (WES) en Ontario, sont chargés d'évaluer les diplômes des étudiants à leur arrivée au Canada.

Il existe des programmes relais, axés sur un enseignement spécialisé, qui peuvent aider les immigrants à obtenir une licence ou un certificat dans leur profession ou leur métier afin de travailler au Canada. De plus, ils peuvent aider les étudiants à obtenir les compétences dont ils ont besoin ; pas seulement des aptitudes relatives à un travail technique, mais des compétences comportementales, comme l'attitude à adopter sur un lieu de travail. Par exemple, si vous êtes infirmier ou comptable, ces programmes vous aideront à obtenir la reconnaissance de vos études dans la province où vous vivez. Vous pourrez alors travailler dans votre domaine d'études? 


\section{Le paradoxe canadien de l'expérience professionnelle}

C'est l'une des épreuves les plus déroutantes pour les nouveaux arrivants : comment obtenir de l'expérience au Canada si personne ne vous offre l'opportunité de travail ? Il faut savoir qu'au Canada certaines professions sont réglementées afin d'assurer la sécurité et la santé du public comme, par exemple, les médecins, les infirmiers, les ingénieurs, les électriciens et les enseignants. Leurs métiers sont supervisés par un organisme de réglementation. Par exemple, l'Ordre des infirmières et infirmiers de l'Ontario veille à ce que ses membres soient qualifiés pour effectuer le travail d'infirmier. Les organismes de réglementation veillent également à ce que les membres maintiennent leurs qualifications et satisfassent certaines normes de pratique $^{8}$.

Ces organismes sont mandatés pour : définir les conditions d'admission et de formation, définir des normes de bonne pratique; évaluer les compétences, reconnaître les attestations d'études, la formation ou l'expérience ; inscrire les demandeurs qualifiés ; émettre les permis ou certificats relatifs aux emplois dans les professions réglementées ; discipliner les membres.

Officiellement, les professions réglementées ne représentent que $20 \%$ des professions canadiennes. Donc 1 nouvel arrivant sur 5 aura besoin d'un permis ou d'une licence. Mais, dans la réalité, cette proportion est plus élevée, car la catégorie la plus importante des immigrants au Canada est celle "des travailleurs qualifiés et professionnels" (environ 60 à $70 \%$ de tous les immigrants). Selon les règles de l'immigration, ces personnes doivent faire partie des catégories suivantes : genre de compétence 0 (postes de gestion) ou niveau de compétence A (emplois professionnels) ou B (emplois techniques et spécialisés). Cette classification nationale des professions ${ }^{9}$ montre que la majorité de ces profes- sions sont réglementées! En somme, beaucoup d'immigrants sénégalais sont confrontés à cette barrière de la réglementation.

Si beaucoup de gens s'installent dans des lieux qui facilitent leur communication, en étant proches de leur famille ou d'autres immigrants qui viennent du même endroit qu'eux, arrive un moment où tout nouvel arrivant a besoin de sortir de sa zone de confort pour construire sa situation professionnelle. La maitrise de l'anglais représente un pas important dans le processus d'établissement, car elle permet de s'impliquer dans des événements, de participer, de voter avec confiance. Cela aide également à obtenir les meilleurs emplois qui, au Québec, sont bilingues.

L'immigrant diplômé sénégalais est toujours confronté à cette barrière lors des différentes entrevues dans les grandes entreprises, en particulier à Montréal. Sans la

\author{
L'insertion des étudiants \\ diplômés qui arrivent \\ au Canada n'est pas chose \\ facile. Il faut compter au moins \\ cing ans avant de pouvoir \\ trouver un emploi en accord \\ avec sa spécialité.
} pas les mêmes opportunités que les autres Canadiens. Selon Statistique Canada, le recensement de 2006 a montré que $80 \%$ de la population ont l'anglais ou le français pour langue maternelle (respectivement $58 \%$ et $22 \%) .98 \%$ de la population peuvent parler une ou même les deux langues officielles. En outre, l'anglais ou le français est parlé au moins régulièrement à la maison par $94 \%$ des Canadiens. Ils constituent les langues de communication à la maison pour $89 \%$ de la population, parfois en combinaison avec une langue non officielle.

\section{Conclusion}

L'insertion des étudiants diplômés qui arrivent au Canada prend au moins cinq ans. C'est la période nécessaire pour qu'ils trouvent un emploi en accord avec leurs spécialités. La plupart des étudiants sénégalais diplômés s'installent au Québec, 
en particulier à Montréal, où ils doivent faire face à la concurrence des autres immigrants. Cependant, pour outrepasser ce délai de cinq ans, l'immigrant peut accepter d'aller en région, c'est-à-dire en dehors de Montréal, ou dans une autre province du Canada. Afin de lutter contre le chômage des diplômés, le gouvernement du Québec a mis sur pied un programme pour promouvoir l'installation dans les régions. Cependant, elles restent peu attractives pour beaucoup de diplômés, et c'est le cas de la majorité des étudiants sénégalais diplômés venus de l'extérieur du pays.

Enfin, le Sénégal a adopté un nouveau modèle de développement pour accélérer son développement économique : le plan Sénégal émergent (PSE), mis en place en novembre 2012 par le gouvernement et l'ensemble de ses partenaires. L'ambition du Sénégal est de favoriser la croissance économique à fort impact sur le développement humain. Or, parmi les étudiants sénégalais établis au Canada, leur pays d'origine dispose d'un énorme bassin de recrutement de personnes hautement qualifiées. Cette catégorie de travailleurs profite de son installation permanente au Canada et gagne en expérience en travaillant dans des milieux qui stimulent leur savoir-faire. Reste à savoir si le Sénégal se donne les moyens de reconnecter avec cette diaspora intellectuelle. Comment faire pour convaincre ces travailleurs hautement qualifiés de participer au développement de leur pays d'origine? L'État sénégalais fait face au défi d’engager sa diaspora au Canada dans le développement du pays ${ }^{10}$.

L'Europe a toujours été la destination majeure des Sénégalais dans le cadre de la migration internationale. D'après le rapport sur la migration au Sénégal, Profil national, publié en 2009, le nombre de Sénégalais immatriculés en Europe représente $66 \%$ des Sénégalais à l'extérieur. Ce chiffre est à considérer avec beaucoup de circonspection, en raison des divergences entre les sources nationales et internationales. Cependant, depuis une décennie, avec la crise que traversent certains pays d'Europe, on constate une re-émigration des Sénégalais vers d'autres pays. C'est le cas des étudiants Sénégalais en Europe : faute de pouvoir s'intégrer dans leurs pays de formation, ils ont fait le choix de ré-émigrer vers le Canada de façon permanente. 